\title{
The Direct Cost of Managing a Rare Disease: Assessing Medical and Pharmacy Costs Associated with Duchenne Muscular Dystrophy in the United States
}

\author{
Sarah Thayer, MA; Christopher Bell, MS; and Craig M. McDonald, MD
}

\begin{abstract}
BACKGROUND: A Duchenne muscular dystrophy (DMD) cohort was identified using a claims-based algorithm to estimate health care utilization and costs for commercially insured DMD patients in the United States. Previous analyses have used broad diagnosis codes that include a range of muscular dystrophy types as a proxy to estimate the burden of DMD.

OBJECTIVE: To estimate DMD-associated resource utilization and costs in a sample of patients identified via a claims-based algorithm using diagnosis codes, pharmacy prescriptions, and procedure codes unique to DMD management based on DMD clinical milestones.

METHODS: DMD patients were selected from a commercially insured claims database (2000-2009). Patients with claims suggestive of a non-DMD diagnosis or who were aged 30 years or older were excluded. Each DMD patient was matched by age, gender, and region to controls without DMD in a 1:10 ratio (DMD patients $n=75$; controls $n=750$ ). All-cause health care resource utilization, including emergency department, inpatient, outpatient, and physician office visits, and all-cause health care costs were examined over a minimum 1-year period. Costs were computed as total health-plan and patientpaid amounts of adjudicated medical claims (in annualized U.S. dollars).
\end{abstract}

RESULTS: The average age of the DMD cohort was 13 years. Patients in the DMD cohort had a 10-fold increase in health care costs compared with controls $(\$ 23,005$ vs. $\$ 2,277, P<0.001)$. Health care costs were significantly higher for the DMD cohort across age strata and, in particular, for DMD patients aged $14-29$ years $(\$ 40,132$ vs. $\$ 2,746, P<0.001)$.

CONCLUSIONS: In the United States, resource use and medical costs of DMD are substantial and increase with age.

J Manag Care Spec Pharm. 2017;23(6):633-41

Copyright $\odot 2017$, Academy of Managed Care Pharmacy. All rights reserved.

\section{What is already known about this subject}

Duchenne muscular dystrophy (DMD) is a severe, progressive disease that occurs in approximately 1 in 3,500-6,000 live male births. The management of DMD is resource intensive and places a substantial burden on patients, families, and health care systems.

\section{What this study adds}

This claims-based study identifed a cohort of DMD patients using an algorithm rather than a single, broad muscular dystrophy diagnosis code

Study results showed that annual health care costs for DMD patients were roughly 10 times higher $(>\$ 20,000)$ per patient compared with individuals without DMD; total costs were also significantly higher for older DMD patients after the disease progressed and care and resource use intensified.

This study provides insight into how health care utilization and costs differ for DMD patients over the course of their disease and will help evaluate current costs of DMD care and incremental costs of new therapies as they are developed.

1 uscular dystrophies are a heterogeneous group of neuromuscular diseases, marked by progressive wasting and weakness of the muscle and other tissues. They vary by gene involved, inheritance pattern, age of onset, and severity and distribution of muscle involvement. ${ }^{1,2}$ Duchenne muscular dystrophy (DMD) is the most common form of the inherited muscular dystrophies. It is an X-linked recessive disorder characterized by mutations in the dystrophin gene located at Xp21, resulting in a defect or near complete absence of the protein dystrophin. The prevalence of DMD has been estimated at between 1 in 3,500-6,000 live male births. ${ }^{3-5}$

This incurable disease almost exclusively affects young males. Complete loss of dystrophin causes increased muscle fragility, and males with dystrophinopathy experience progressive loss of muscle fibers from early infancy, with replacement of fibers by fat and connective tissue, severe disabling weakness, contractures and scoliosis, loss of ambulation and self-care skills, respiratory and cardiac failure, and premature death. ${ }^{6}$ The average age at DMD diagnosis is 5 years. ${ }^{7}$ Although signs of delayed and abnormal motor development in affected boys begin in early childhood, there is a delay of about 2.5 years between onset of DMD symptoms and the time of definitive diagnosis. ${ }^{8}$ Affected individuals typically begin with mildly delayed motor milestones and inability to run or jump properly. Loss of standing ability typically occurs in early childhood, with loss of ambulation before adolescence, and 
orthopedic, respiratory, and cardiac complications occur as the disease progresses during adolescence and the early twenties. In the natural history of the disease, the average age at death is 19 years. $^{7}$ The severity of this neuromuscular disorder makes patients largely dependent on caregivers, who are typically their parents, throughout life. ${ }^{6}$

Improved medical management is leading to longer life spans. Improvements in multidisciplinary disease management, including corticosteroid treatment to improve muscle strength and prolong ambulation, surgical management of scoliosis, and timely respiratory and cardiac interventions, have led to a profound increase in life expectancy. For example, 2 studies have demonstrated a mean 8 - to 10 -year prolongation in survival among those DMD patients managed with noninvasive ventilation compared with those not ventilated. ${ }^{9,10}$ Most children diagnosed today may live into their fourth decade with noninvasive ventilation and optimal cardiac management. ${ }^{7,10}$ However, with improved survival there is also a longer lived burden of disease.

While there are no regulatory-approved treatments that change the natural course of the disease, glucocorticoids are considered the gold standard treatment in ambulant children with DMD. ${ }^{7}$ Studies of glucocorticoids have demonstrated their ability to slow decline in muscle strength and function, ${ }^{11-13}$ with some long-term studies demonstrating prolongation of function and ambulation by 2-3 years and other studies supporting early initiation for improved outcomes. ${ }^{14-17}$ Furthermore, continued use of steroids after loss of ambulation has positive effects on preserving upper limb strength, ability to self-feed, and delaying decline in respiratory and cardiac function. ${ }^{11,12,15,16,18}$ Glucocorticoid treatment has also resulted in a lower percentage of patients developing scoliosis. ${ }^{18}$

Early monitoring is indicated to detect asymptomatic cardiomyopathy. In the presence of progressive abnormalities, such as left ventricular dysfunction, medications (e.g., angiotensin-converting enzyme (ACE) inhibitors and beta blockers) are initiated to control symptoms and prevent deterioration to heart failure. ${ }^{19-21}$ Early prophylactic treatment with an ACE inhibitor is associated with improved survival. ${ }^{20}$ To treat respiratory complications, studies have demonstrated positive response to ventilation, either noninvasive or by tracheostomy. ${ }^{22}$ For instance, the mean age of death rose from 19 years to at least 25 years with the use of nocturnal ventilation in a United Kingdom study. ${ }^{23}$

The management of DMD is resource intensive and places a substantial burden on patients, their families, and health care systems. Few studies have quantified the cost of care specifically for DMD in the United States. A U.S. study by Ouyang et al. (2008) estimated medical resource utilization and expenditures for privately insured children and young adults (aged $<30$ years) with any form of muscular dystrophy. ${ }^{2}$ They estimated annual average expenditures of medical care to be $\$ 20,467$ in 2004, 13 times higher than individuals without muscular dystrophy. Not surprisingly, the difference in mean expenditures between individuals with muscular dystrophy (MD) and without MD increased with age, from $\$ 13,464$ at ages 5-9 years to $\$ 32,541$ at ages $15-19$ years. ${ }^{2}$ More recently, Larkindale et al. (2014) used U.S. commercial and Medicare claims data to estimate direct medical costs and a cost of illness survey to estimate nonmedical costs (e.g., costs for modifications to houses and vehicles to accommodate the affected individual) and loss of income associated with neuromuscular diseases. ${ }^{24}$ The study focused on 3 diseases-amyotrophic lateral sclerosis, myotonic dystrophy, and DMD - and found that the total annual per-patient medical cost (including paid insurance and out-of-pocket amounts) was $\$ 22,533$, and the total nonmedical cost was $\$ 12,939$ for DMD in 2010 .

To date, however, no U.S. study has been able to define or provide accurate costs of the specific DMD phenotype of the disease because of the lack of a specific diagnosis code Previous studies have relied on using diagnosis codes as proxies to define DMD, which would include other less severe forms of MD, such as Becker muscular dystrophy (BMD) and Limb-Girdle muscular dystrophy (LGMD). For example, Larkindale et al. used the International Classification of Diseases, Ninth Revision, Clinical Modification (ICD-9-CM) diagnosis code 359.1 (hereditary progressive muscular dystrophy) as a proxy. ${ }^{24}$ Better ways to estimate the cost of managing this severe form of muscular dystrophy in the United States are needed.

The objective of this study was to estimate patient-level health care utilization and costs associated with DMD by identifying DMD patients via a claims-based algorithm using diagnosis codes, pharmacy prescriptions, and procedure codes unique to DMD management and consistent with clinical milestones stereotypical for DMD. This algorithm will better identify patients with DMD, which will enable more specific estimates of the health care expenditures associated with DMD.

\section{Methods}

\section{Study Design and Data Sources}

This was a retrospective analysis of medical claims, pharmacy claims, and enrollment information from a large, national U.S. health plan affiliated with Optum. The individuals covered by this health plan, about 13 million annual commercially insured members in 2009, were geographically diverse across the United States, with greatest representation in the South and Midwest census regions. Medical (professional, facility) claims included ICD-9-CM diagnosis codes, ICD-9-CM procedure codes, Current Procedural Terminology (CPT), 4th edition, procedure codes, Healthcare Common Procedure Coding System procedure codes, site of service codes, provider specialty codes, and health plan and patient costs (Appendix, 


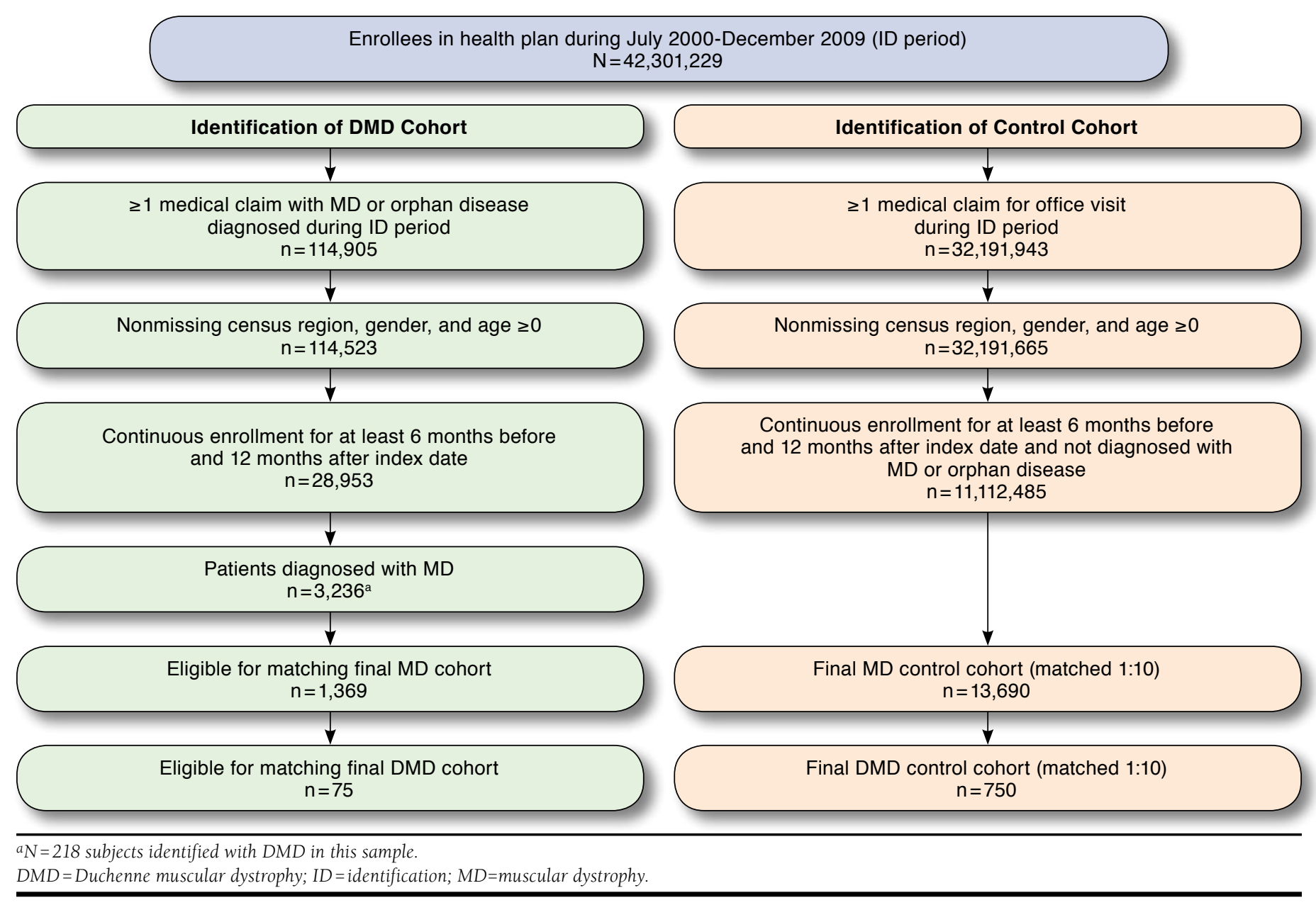

available in online article). The total period of observation for this study was January 1, 2000-December 31, 2010.

Claims data were accessed in compliance with the Health Insurance Portability and Accountability Act. ${ }^{25}$ Identifiable patient information was not included in the dataset analyses. This study was approved by a privacy board and an institutional review board.

\section{Study Population}

Sample selection is summarized in Figure 1. Commercially insured health plan members were eligible for the study if they had at least 1 medical claim with a primary or secondary diagnosis for MD (ICD-9-CM diagnosis code 359.0 [congenital hereditary muscular dystrophy] or 359.1 [hereditary progressive muscular dystrophy] between July 1, 2000, and December 31, 2009 (identification period). The service date of the first observed claim for MD was defined as the index date for patients in the DMD cohort.
To be included in the control cohort, patients must have had at least 1 medical claim for an office visit during the identification period and no medical claims with diagnosis codes for MD (ICD-9-CM codes 359.0 or 359.1) or the following rare diseases: lipid storage disorders (ICD-9-CM code 272.7), mucopolysaccharidosis (ICD-9-CM code 277.5), cystic fibrosis (ICD-9-CM code 277.0x), or myasthenia gravis (ICD-9-CM code $358.0 x)$ at any time during the study period. The index date for the control cohort was assigned to a randomly chosen office visit during the identification period. All subjects must have been continuously enrolled in the health plan with medical and pharmacy benefits for 182 days before their index date and for at least 365 days after their index date.

\section{DMD Cohort Assignment}

A claims-based algorithm was used to identify probable DMD patients by using diagnosis codes, pharmacy prescriptions, or 


\begin{tabular}{|c|c|c|c|c|c|}
\hline & \multicolumn{2}{|c|}{$\begin{array}{l}\text { DMD Cohort } \\
\quad(\mathrm{n}=75)\end{array}$} & \multicolumn{2}{|c|}{$\begin{array}{l}\text { Matched Control Cohort } \\
\qquad(\mathrm{n}=750)\end{array}$} & \multirow[b]{2}{*}{$P$ Value } \\
\hline & $\mathrm{n}$ & $\%$ & $\mathrm{n}$ & $\%$ & \\
\hline Age (continuous), years & 13.11 & 6.88 & 13.11 & 6.84 & \multirow[t]{6}{*}{1.000} \\
\hline $0-3$ & 4 & 5.33 & 40 & 5.33 & \\
\hline $4-7$ & 14 & 18.67 & 140 & 18.67 & \\
\hline $8-13$ & 23 & 30.67 & 230 & 30.67 & \\
\hline $14-17$ & 20 & 26.67 & 200 & 26.67 & \\
\hline $18-29$ & 14 & 18.67 & 140 & 18.67 & \\
\hline Male & 75 & 100.00 & 750 & 100.00 & 1.000 \\
\hline \multicolumn{5}{|l|}{ Geographic region } & 1.000 \\
\hline Northeast & 3 & 4.00 & 30 & 4.00 & \\
\hline Midwest & 26 & 34.67 & 260 & 34.67 & \\
\hline South & 32 & 42.67 & 320 & 42.67 & \\
\hline West & 14 & 18.67 & 140 & 18.67 & \\
\hline \multicolumn{6}{|l|}{ Provider specialty on index date } \\
\hline Primary care/family practice/pediatrics/internal medicine & 7 & 9.33 & 519 & 69.2 & $<0.001$ \\
\hline Neurology & 15 & 20.00 & 4 & 0.53 & $<0.001$ \\
\hline Cardiology & 0 & 0.00 & 1 & 0.13 & 0.752 \\
\hline Hospital/other/unknown & 53 & 70.67 & 226 & 30.14 & $<0.001$ \\
\hline Number of days follow-up (mean, SD) & $1,071.83$ & 794.80 & 888.51 & 549.95 & 0.054 \\
\hline
\end{tabular}

procedures unique to DMD management and consistent with clinical milestones stereotypical for DMD. To be included in the DMD cohort, patients were required to meet the previously listed criteria for MD, be male, and at any time during the study period have had at least 1 of the following:

- At least 1 medical claim for evidence of wheelchair use at age $\leq 16$ years.

- At least 1 pharmacy claim for corticosteroid use at age $\geq 4$ years. $^{\top}$

- If evidence of steroid treatment, at least 1 medical claim for evidence of wheelchair use at age $\geq 16$ years but $\leq 19$ years.

- At least 1 medical claim for cardiomyopathy (ICD-9-CM diagnosis codes 425 . $x$ and $674.5 x)$ at age $\geq 6$ years.

- At least 1 pharmacy claim for ACE inhibitors or angiotensin II receptor blockers at age $\leq 18$ years.

- At least 1 medical claim for ventilator, respiratory failure, or respiratory therapy (see Appendix).

- At least 1 medical claim any time during an inpatient stay with a V code for terminal illness (ICD-9-CM V66.7 encounter for palliative care) at age $\geq 17$ years.

Patients were excluded from the DMD cohort if they had 2 or more medical claims for ventilator use separated by at least 180 days before age 6 years; at least 1 medical claim with a CPT code for an orthopedic procedure on the foot before age 4 years (common in congenital myotonic dystrophy or several forms of congenital MD [CMD]); at least 1 medical claim for a power wheelchair before age 6 years (to exclude patients with CMD or autosomal recessive forms of LGMD); or if they were aged 30 years or greater at any time during their pre-index or post-index period. The final exclusion was made post hoc because, although DMD patients may live beyond age 30, the proportion of MD patients with a non-DMD ICD-9-CM diagnosis is much higher after age 30 .

Patients in the DMD cohort were matched to the healthy controls in a 1:10 ratio on age, gender, and geographic region.

\section{Variables}

Patient variables included age; gender; geographic region (Northeast, Midwest, South, and West); number of days of follow-up; and provider specialty on the index date. Clinical characteristics were measured in the 6-month pre-index period and included Quan-Charlson comorbidity score, medications, health care utilization, and costs.

During the post-index period, we examined all-cause health care resource utilization including emergency department (ED), inpatient, outpatient, and physician office visits and allcause health care costs. Costs were computed as total healthplan and patient-paid amounts of adjudicated medical claims (in U.S. dollars). Costs were adjusted to 2010 using the annual medical care component of the Consumer Price Index to reflect inflation between 2000 and 2010.26

\section{Statistical Analysis}

Patient characteristics were compared between the matched cohorts using McNemar's test for paired data for categorical measures and paired t-tests for continuous 
The Direct Cost of Managing a Rare Disease: Assessing Medical and

Pharmacy Costs Associated with Duchenne Muscular Dystrophy in the United States

TABLE 2 Post-index Health Care Resource Utilization and Costs (Annualized, Unadjusted)

\begin{tabular}{|c|c|c|c|c|c|}
\hline & \multicolumn{2}{|c|}{$\begin{array}{l}\text { DMD Cohort } \\
\quad(\mathrm{n}=75)\end{array}$} & \multicolumn{2}{|c|}{$\begin{array}{l}\text { Matched Control Cohort } \\
\qquad(\mathrm{n}=750)\end{array}$} & \multirow[b]{2}{*}{$P$ Value } \\
\hline & Mean & SD & Mean & SD & \\
\hline \multicolumn{6}{|l|}{ All-cause health care utilization } \\
\hline Office visit & 11.92 & 16.92 & 4.50 & 5.25 & $<0.001$ \\
\hline Outpatient visit & 6.42 & 6.39 & 0.84 & 1.79 & $<0.001$ \\
\hline Inpatient admission & 0.23 & 0.47 & 0.02 & 0.14 & $<0.001$ \\
\hline Emergency room visit & 0.68 & 1.22 & 0.31 & 1.27 & 0.016 \\
\hline Unique medications, per year & 2.95 & 2.47 & 1.54 & 1.65 & $<0.001$ \\
\hline Medication dispensings, per year (Rx fills) & 16.31 & 18.81 & 3.93 & 5.55 & $<0.001$ \\
\hline \multicolumn{6}{|l|}{ All-cause health care costs (U.S. \$) } \\
\hline Ambulatory & 4,838 & 5,105 & 1,015 & 2,049 & $<0.001$ \\
\hline Inpatient & 11,274 & 33,859 & 313 & 2,514 & 0.006 \\
\hline Emergency room & 517 & 1,706 & 60 & 184 & 0.023 \\
\hline Other $^{\mathrm{a}}$ & 5,901 & 9,695 & 63 & 313 & $<0.001$ \\
\hline Medical cost & 22,530 & 39,041 & 1,451 & 3,745 & $<0.001$ \\
\hline Pharmacy cost & 1,487 & 2,861 & 301 & 663 & $<0.001$ \\
\hline Total cost & 24,017 & 39,369 & 1,752 & 3,870 & $<0.001$ \\
\hline
\end{tabular}

${ }^{a}$ Other costs defined as anything not classified as ambulatory, inpatient or emergency room; includes items such as durable medical equipment and ambulance.

$R x=$ prescription; $S D=$ standard deviation.

measures. Annualized post-index total health care costs in the DMD and control cohorts were compared using a multivariate generalized linear model with a gamma distribution and log link. Estimated cost measures were modeled using Blough et al.'s formulation (1999). ${ }^{27}$ For ease of interpretation and comparison with the bivariate results, the average cost was predicted for each cohort. Cost ratios, 95\% confidence intervals, and $P$ values were presented for each covariate of interest (i.e., DMD and control cohorts) included in the final model. Because of small sample size, age groups were combined and cost models were completed for ages 0-13 years and $14-29$ years.

\section{Results}

During the identification period, 144,905 patients had at least 1 medical claim with a diagnosis code for MD or other rare diseases of interest, including lipid storage disorders, mucopolysaccharidosis, cystic fibrosis, or myasthenia gravis (analyzed separately). Removal of patients with missing region, gender, and age $<0$, less than 6 months continuous enrollment before the index date, and less than 1 year continuous enrollment after the index date left 3,236 patients with MD. Of these, 218 patients were classified as DMD, and 75 DMD patients were matched 1:10 to 750 control subjects in the final cohorts.

Patient baseline characteristics are presented in Table 1. As shown, patients in the DMD cohort were all male and aged under 30 years. Almost $60 \%$ of the DMD cohort was aged between 8 and 17 years, with an average of 13 years. DMD patients primarily resided in the South (43\%) and Midwest (35\%) regions, generally in line with the geographic distribution of the health plan enrollment. Most patients in the DMD cohort $(71 \%)$ were seen at the hospital by an unknown provider type or other on the index date, whereas most patients in the control cohort (69\%) were seen by a primary care provider (including pediatrics and internal medicine) on the index date. Compared with controls, patients in the DMD cohort remained enrolled in the health plan for a greater number of days in the follow-up period (1,072 days vs. 889 days).

Table 2 compares the annualized post-index health care resource utilization and costs for patients in the DMD cohort with matched controls. DMD patients had statistically significantly higher unadjusted health care utilization, including twice as many office visits, 6 times as many outpatient visits, 10 times as many inpatient stays, and 4 times as many prescription fills per year. Annual medical costs for the DMD cohort were $\$ 22,530$ compared with $\$ 1,451$ for the control cohort, a difference of $\$ 21,079$ per year. Annual pharmacy costs were $\$ 1,186$ higher for DMD patients. The higher medical and pharmacy costs for DMD patients resulted in a difference in total costs of over $\$ 22,000$.

Figure 2 shows the post-index all-cause health care utilization for the DMD and matched control cohorts stratified by age category. In all age groups, DMD patients had 6- to 8-fold increases in outpatient visits as matched controls $(P<0.05$ for all age groups), with the greatest difference in the 14-17 years age group. In the 8-13 years age group, office visits (8.92 vs. $4.44, P=0.047)$ and outpatient visits (6.72 vs. $0.70, P<0.001)$ were higher. In the 14-17 years age group, office visits (8.99 vs. $4.73, P=0.003)$, outpatient visits ( 8.52 vs. $1, P<0.001$ ), and inpatient stays $(0.47$ vs. $0.03, P=0.008)$ were higher. In the $18-29$ years age group, office visits $(12.71$ vs. $3.73, P=0.007)$ and outpatient visits (3.50 vs. $1.04, P=0.004$ ) were higher. 


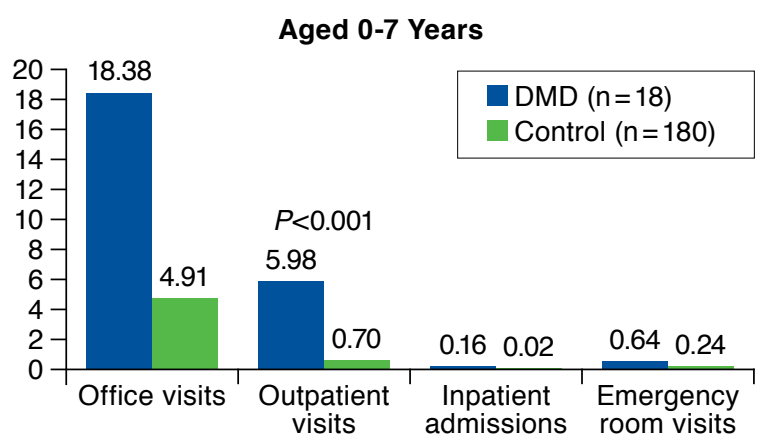

Aged 14-17 Years

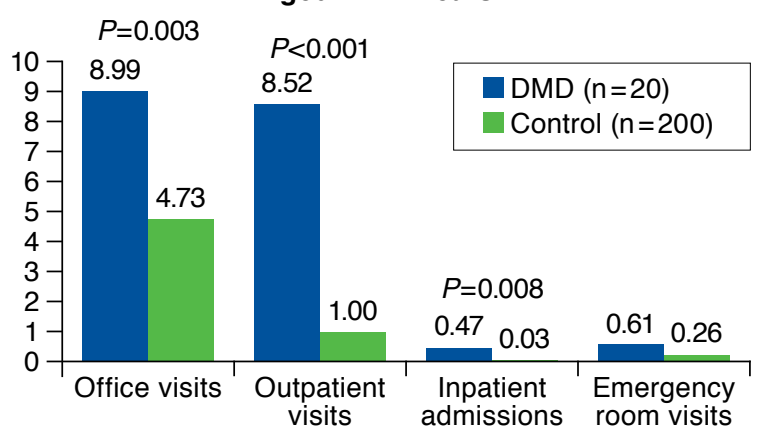

Aged 8-13 Years

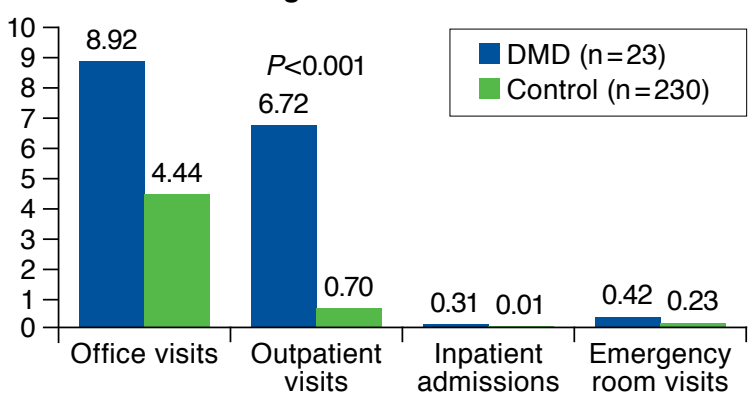

Aged 18-29 Years

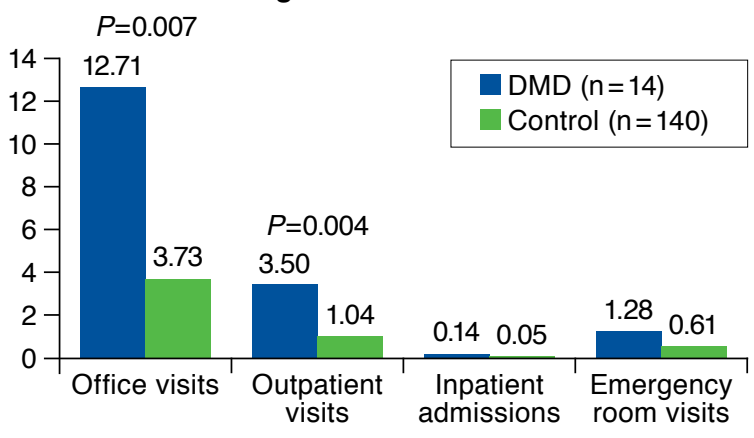

DMD = Duchenne muscular dystrophy.

Results from a generalized linear model of annualized total health care costs among all patients in the DMD cohort estimated a 10-fold increase in health care costs compared with matched controls ( $\$ 23,005$ vs. $\$ 2,277, P<0.0001$; Figure 3 ). Total health care costs were statistically significantly higher for the DMD cohort across age strata, particularly for DMD patients aged $14-29$ years ( $\$ 40,132$ vs. $\$ 2,746, P<0.0001)$.

\section{Discussion}

$\overline{\text { This analysis of health plan administrative data allowed for }}$ estimation of real-world patient-level health care utilization and costs between a cohort of DMD patients and a cohort of matched controls. Compared with the control group, the DMD group had a 10 -fold increase in health care cost $(\$ 23,005$ vs. $\$ 2,277, P<0.0001$ ), mainly because of higher medical cost (difference of $\$ 22,000$ ), which illustrates the high intensity of resources required to manage this debilitating disease. We also found that medical need and health care resource utilization intensifies as DMD progresses, with a corresponding increase in costs, especially after age 14 years. Higher costs during the older ages were primarily attributable to a greater number of outpatient visits and office visits. This is consistent with what is known about the natural course of the disease-as DMD progresses, loss of ambulation and increased respiratory difficulties require an increase in intensity of care and resources. Results of a nationwide survey conducted in the United Kingdom between April and June 2010 found that patients and their families living with neuromuscular conditions face extreme financial hardship, primarily because of the cost of assistive devices and home modifications, as well as lost wages. ${ }^{28}$

Our findings are closely aligned with other estimates of medical care utilization and expenditures for U.S. insured populations. Ouyang et al. found that in 2004 the average medical expenditures for commercially insured individuals with MD were also high at $\$ 20,467$ per year ${ }^{2}$; however, these estimates were not specific to DMD patients, since ICD-9-CM diagnosis codes 359.0 (congenital hereditary muscular dystrophy) and 359.1 (hereditary progressive muscular dystrophy) — which include diagnosis of DMD and other forms of MD-were used to identify study subjects. In another study, Larkindale et al. estimated the total annual per-patient medical cost for DMD in 2010 (weighted by proportion of patients covered by the different insurance types) at $\$ 22,533 . .^{24}$ The population was selected using the ICD-9-CM code 359.1 (hereditary progressive muscular dystrophy) as a proxy for DMD, which likely underestimated the costs of patients with DMD, since less severe forms of MD (e.g., BMD and LGMD) are included under the 359.1 code. 
FIGURE 3 Average Total Health Care Costs for DMD and Matched Control Cohorts

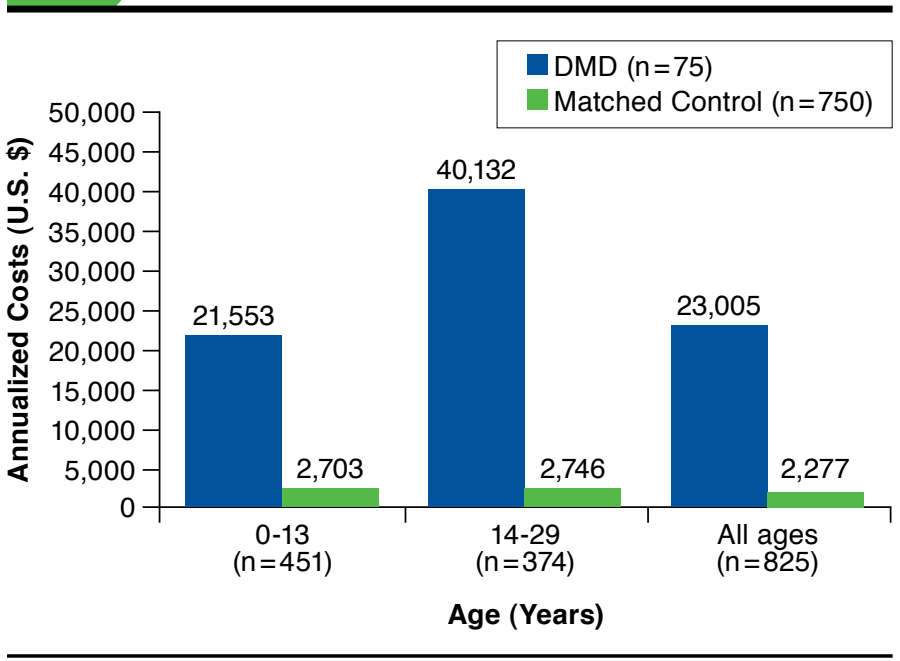

DMD = Duchenne muscular dystrophy.

In this study, we attempted to identify a DMD cohort using an algorithm rather than a single, broad ICD diagnosis code that includes a range of MD types that differ by inheritance pattern, age of onset, disease severity, and rate of progression. As a result, the algorithm was more likely to include potential patients with DMD compared with those just selected by the ICD-9-CM diagnosis code, but validation of this algorithm in future studies is warranted. A diagnosis code developed specifically for DMD would help differentiate patients with DMD from other forms of MD, which would allow a better estimation of health care utilization and costs incurred by patients with DMD.

There are many promising novel therapeutic strategies for DMD that have been evaluated in recent clinical trials. In addition to corticosteroids, which ameliorate disease progression in DMD and have increasingly become the standard of care since 2010, ${ }^{29,30}$ dystrophin restoration therapies represent a clear advance in treating the underlying cause of DMD, with the goal being the restoration of low levels of dystrophin to transition patients from a DMD phenotype to a more slowly progressive phenotype similar to BMD. Thirteen percent of patients have a nonsense or premature stop codon mutation. Ataluren (marketed as Translarna in the European Union) binds to the ribosome and induces read-thru of a premature stop codon mutation and low levels of dystrophin. In ambulatory DMD patients aged 5 years and older, ataluren has been shown to slow disease progression as measured with the 6-minute walk distance (6MWD), and it also slowed the deterioration of function across timed function tests. ${ }^{31}$ In 2014, the European Medicines Agency provided a conditional approval for Translarna, representing the first approved treatment of the underlying cause of dystrophinopathy for a subpopulation of DMD patients.
Alternatively, exon skipping strategies use either antisense oligonucleotide or phosphorodiamidate morpholino oligomer (PMO) chemistries to bind to mRNA and induce exon skipping with restoration of the open-reading frame of the dystrophin gene and a production of a shortened but functional dystrophin protein. Exon skipping strategies could theoretically treat up to $70 \%$ of all DMD patients, and many drugs are in the therapeutic pipeline that target specific genetic constructs among subpopulations of DMD patients. Eteplirsen is a PMO chemistry that skips exon 51 and appears to have a favorable safety profile. Results of a phase 2 study of weekly intravenous infusions of eteplirsen in 10 patients with higher baseline 6MWD showed dystrophin production by 24 weeks and decreased disease progression measured by 6MWD at 3 years. ${ }^{32}$ The U.S Food and Drug Administration recently provided accelerated approval for eteplirsen (Exondys 51) in DMD partients with exon 51 amenable mutations for exon skipping. A confirmatory trial of eteplirsen is pending. It remains to be seen what the additional effect of these and other novel therapeutics on the horizon will have on the burden and cost of DMD.

Consensus care considerations for DMD using expert opinions and RAND methodology were first published in 2010 by Bushby et al. ${ }^{7,33}$ These care considerations have had an effect on the standards of care currently provided to DMD patients and the nature of the multidisciplinary expert care provided in order to optimally manage the disease. Our study used claims data from a period of time before the publication of these care standards. It will be important to perform follow-up analyses focusing on the effect of these new emerging care standards for DMD on the burden of disease and costs of patient care.

\section{Limitations}

The strength of our analysis derives from the large, geographically diverse population studied and suggests that health care utilization and costs are substantially higher in DMD patients, particularly those who are teenagers and older. However, it is recognized that these findings must be interpreted with appropriate consideration of limitations inherent to claims analyses.

First, the presence of a medical or pharmacy claim does not necessarily indicate that a service or drug was administered. Second, claims data are collected primarily for payment purposes, not research, and are subject to coding errors. Third, the lack of a specific ICD-9-CM diagnosis code for DMD makes case ascertainment less certain. Future research will encounter similar challenges, since the new ICD-10 coding system has the same limitations.

Although the case ascertainment algorithm used in this study was developed in partnership with an expert clinician using codes consistent with typical DMD clinical milestones, the presence of a diagnosis code for MD on a medical claim, combined with our DMD case ascertainment algorithm, is not positive presence of DMD. As with the series by Larkindale et al., ${ }^{24}$ 
our estimates may be undervalued because of the potential dilution of the proxy code by BMD and less severe forms of MD. While our criteria for DMD addressed this concern to a large degree, there is a possibility that costs in a clinically confirmed DMD cohort could be even higher.

Another limitation stems from the inclusion requirement that individuals have at least 18 months of continuous health plan enrollment. Our results are generalizable to patients in stable managed care settings but not to those with no insurance coverage or with Medicaid or Medicare fee for service, which may equate to roughly $49 \%$ of DMD patients. ${ }^{24}$ Also, the inclusion requirement of continuous enrollment for 12 months post-index may have excluded some terminally ill patients who died during the year, leading to underestimates of health care utilization and expenditures. In addition, nonmedical care and indirect costs, such as out-of-pocket expenditures for modifications to the home, costs of paid and unpaid caregiving, and productivity losses, were not included in this study and may have resulted in an underestimate of the true medical costs.

Finally, it is important to remember that the burden of DMD goes beyond medical resource utilization. Access to pulmonary durable medical equipment, such as airway clearance devices, suction apparatuses, and noninvasive sip and puff ventilation devices interfaced with the wheelchair may reduce complications. In addition, a host of other assistive devices, such as electric beds, alternating pressure relieving mattresses, patient lifts, custom shower and toilet chairs, power wheelchairs, pressure relieving cushions, mobile arm supports, upper extremity robotics, environmental control units, computer access interfaces, automotive access lifts and ramps, and other adaptations to housing are essential to ease the burden of this devastating disease for patients and their caregivers.

Through their cost-of-illness survey, Larkindale et al. estimated that the total per-patient annual nonmedical costs for DMD in 2010 were $\$ 12,939$, and the total indirect costs were $\$ 15,481$, bringing their overall estimated societal cost of DMD to $\$ 50,952$ per year. ${ }^{24}$ An Australian cost of illness study estimated that in 2005 the annual financial cost of various forms of MD combined (DMD, BMD, CMD, LGMD, facioscapulohumeral, myotonic, and distal) was $\$ 126,000$ AUS per person with MD. ${ }^{28}$ This cost was primarily a result of productivity losses (54.2\%); informal care (27.1\%); government payments and forgone taxation (9.7\%); and indirect costs, such as aids and home modifications, formal care services, transport and the bring-forward of funeral costs (6.8\%), while only $2.2 \%$ of the total cost was a result of direct health system expenditures. ${ }^{34}$ Results of a nationwide survey conducted in the United Kingdom between April and June 2010 found that patients and their families living with neuromuscular conditions face extreme financial hardship, primarily because of the cost of assistive devices and home modifications, as well as lost wages. ${ }^{28}$
Despite these limitations, this study provides an accurate understanding of the health care utilization and expenditures specifically for patients with DMD in a real-world U.S. setting compared with matched controls. These findings will help researchers track how expenditures change over time as standards of care and medications are developed. Additional research could include a chart review to validate this newly created claims-based algorithm to confirm that this methodology more accurately identifies patients with DMD. Follow-up analyses focusing on the impact of new emerging care standards for DMD, including new medications as they are developed, will be needed to understand the burden of disease and costs of patient care.

\section{Conclusions}

In the United States, the health care utilization and medical costs of DMD are substantial and increase with age. The increased survival of DMD with ventilation and more effective cardiac management will likely be associated with increased medical costs in the future.

\section{Authors}

SARAH THAYER, MA, Optum Health Economics and Outcomes Research, San Francisco, California; CHRISTOPHER BELL, MS, US Value, Evidence and Outcomes, GlaxoSmithKline, Research Triangle Park, North Carolina; and CRAIG M. MCDONALD, MD, Department of Physical Medicine and Rehabilitation and MDA Neuromuscular Disease Clinics, University of California Davis Health System, Sacramento.

AUTHOR CORRESPONDENCE: Sarah Thayer, MA, Optum, 425 Market St., San Francisco, CA 94105. Tel.: 415.546.3237; E-mail: sarah.thayer@optum.com.

\section{DISCLOSURES}

Funding for this study (GHO-10-4441) was provided by GlaxoSmithKline (GSK). Optum was contracted by GSK to conduct the study. Thayer was an employee of Optum Health Economics and Outcomes Research at the time of this study and was not compensated for her participation as an author of this manuscript. Bell is an employee and shareholder of GSK. McDonald has been a consultant for GSK, Sarepta, PTC Therapeutics, Biomarin, and Catabasis on clinical trials regarding Duchenne muscular dystrophy clinical trial design, endpoint selection, and data analysis; Mitobridge for drug development; and Eli Lilly as part of a steering committee for clinical trials.

Study concept and design were contributed primarily by Bell, along with Thayer and McDonald. Thayer collected the data, and data interpretation was performed by Thayer and Bell, along with McDonald. The manuscript was written by Thayer and Bell, along with McDonald, and revised by all the authors.

\section{ACKNOWLEDGMENTS}

The authors acknowledge the assistance of Megan Coombes and Lourdes Yun for providing medical writing assistance. Coombs was an Optum employee at the time of the writing, and Yun is a current Optum employee. 


\section{REFERENCES}

1. Emery AE. The muscular dystrophies. Lancet. 2002;359(9307):687-95.

2. Ouyang L, Grosse SD, Kenneson A. Health care utilization and expenditures for children and young adults with muscular dystrophy in a privately insured population. J Child Neurol. 2008;23(8):883-88.

3. Emery AE. Population frequencies of inherited neuromuscular diseasesa world survey. Neuromuscul Disord. 1991;1(1):19-29.

4. Mendell JR, Shilling C, Leslie ND, et al. Evidence-based path to newborn screening for Duchenne muscular dystrophy. Ann Neurol. 2012;71(3):304-13.

5. Centers for Disease Control and Prevention. Prevalence of Duchenne/ Becker muscular dystrophy among males aged 5-24 years-four states, 2007. MMWR Morb Mortal Wkly Rep. 2009;58(40):1119-22.

6. Kohler M, Clarenbach CF, Bahler C, Brack T, Russi EW, Bloch KE.

Disability and survival in Duchenne muscular dystrophy. J Neurol Neurosurg Psychiatry. 2009;80(3):320-25.

7. Bushby K, Finkel R, Birnkrandt DJ, et al. Diagnosis and management of Duchenne muscular dystrophy, part 1: diagnosis and pharmacological and pychosocial management. Lancet Neurol. 2010;9(1):77-93.

8. Ciafaloni E, Fox DJ, Pandya S, et al. Delayed diagnosis in duchenne muscular dystrophy: data from the Muscular Dystrophy Surveillance, Tracking, and Research Network (MD STARnet). J Pediatr. 2009;155(3):380-85.

9. Rall S, Grimm T. Survival in Duchenne muscular dystrophy. Acta Myol. 2012;31(2):117-20

10. Passamano L, Taglia A, Palladino A, et al. Improvement of survival in Duchenne muscular dystrophy: retrospective analysis of 835 patients. Acta Myol. 2012;31(2):121-25.

11. Manzur AY, Kuntzer T, Pike M, Swan A. Glucocorticoid corticosteroids for Duchenne muscular dystrophy. Cochrane Database Syst Rev. 2008(1):CD003725.

12. King WM, Ruttencutter R, Nagaraja HN, et al. Orthopedic outcomes of long-term daily corticosteroid treatment in Duchenne muscular dystrophy. Neurology. 2007;68(19):1607-13.

13. Moxley RT 3rd, Ashwal S, Pandya S, et al. Practice parameter: corticosteroid treatment of Duchenne dystrophy: report of the Quality Standards Subcommittee of the American Academy of Neurology and the Practice Committee of the Child Neurology Society. Neurology. 2005;64(1):13-20.

14. Fenichel GM, Florence JM, Pestronk A, et al. Long-term benefit from prednisone therapy in Duchenne muscular dystrophy. Neurology. 1991;41(12):1874-77.

15. Henricson EK, Abresch RT, Cnaan A, et al. The cooperative international neuromuscular research group Duchenne natural history study: glucocorticoid treatment preserves clinically meaningful functional milestones and reduces rate of disease progression as measured by manual muscle testing and other commonly used clinical trial outcome measures. Muscle Nerve. 2013;48(1):55-67.

16. McDonald CM, Han JJ, Mah JK, Carter GT. Corticosteroids and Duchenne muscular dystrophy: does earlier treatment really matter? Muscle Nerve. 2012;45(6):777-79.

17. Merlini L, Gennari M, Malaspina E, et al. Early corticosteroid treatment in 4 Duchenne muscular dystrophy patients: 14-year follow-up. Muscle Nerve. 2012;45(6):796-802.
18. Biggar WD, Harris VA, Eliasoph L, Alman B. Long-term benefits of deflazacort treatment for boys with Duchenne muscular dystrophy in their second decade. Neuromuscul Disord. 2006;16(4):249-55.

19. Bushby K, Straub V. Nonmolecular treatment for muscular dystrophies. Curr Opin Neurol. 2005;18(5):511-18.

20. Duboc D, Meune C, Pierre B, et al. Perindopril preventive treatment on mortality in Duchenne muscular dystrophy: 10 years' follow-up. Am Heart J. 2007;154(3):596-602

21. Schram G, Fournier A, Leduc H, et al. All-cause mortality and cardiovascular outcomes with prophylactic steroid therapy in Duchenne muscular dystrophy. J Am Coll Cardiol. 2013;61(9):948-54

22. Bushby K, Bourke J, Bullock R, Eagle M, Gibson M, Quinby J. The multidisciplinary management of Duchenne muscular dystrophy. Paediatrics and Child Health. 2005;15(4):292-300. Available at: http://www.paediatricsandchildhealthjournal.co.uk/article/S0957-5839(05)00049-7/fulltext. Accessed April 27, 2017

23. Eagle M, Baudouin SV, Chandler C, Giddings DR, Bullock R, Bushby K. Survival in Duchenne muscular dystrophy: improvements in life expectancy since 1967 and the impact of home nocturnal ventilation. Neuromuscul Disord. 2002;12(10):926-29.

24. Larkindale J, Yang W, Hogan PF, et al. Cost of illness for neuromuscular diseases in the U.S. Muscle Nerve. 2014;49(3):431-38.

25. Health Insurance Portability and Accountability Act of 1996, Pub. L. No. 104-191 (1996)

26. U.S. Bureau of Labor Statistics. Chained Consumer Price Index for all urban consumers. C-CPI-U US 1999-2008, medical care. Series ID: SUUR0000SAM. 2008. Available at: http://data.bls.gov/cgi-bin/ surveymost?su. Accessed April 27, 2017.

27. Blough DK, Madden CW, Hornbrook MC. Modeling risk using generalized linear models. J Health Econ. 1999;18(2):153-71.

28. Muscular Dystrophy Campaign. Building on the foundations: the cost of living with muscle disease. 2010. Available at: http://www.musculardystrophyuk.org/app/uploads/2015/03/Cost-of-living-with-muscle-disease.pdf. Accessed May 9, 2017.

29. Henricson EK, Abresch RT, Cnaan A, et al. The cooperative international neuromuscular research group Duchenne natural history study: glucocorticoid treatment preserves clinically meaningful functional milestones and reduces rate of disease progression as measured by manual muscle testing and other commonly used clinical trial outcome measures. Muscle Nerve. 2013;48(1):55-67.

30. Bello L, Gordish-Dressman H, Morgenroth LP, et al. Prednisone/prednisolone and deflazacort regimens in the CINRG Duchenne Natural History Study. Neurology. 2015;85(12):1048-55.

31. Bushby K, Finkel R, Wong B, et al. Ataluren treatment of patients with nonsense mutation dystrophinopathy. Muscle Nerve. 2014;50(4):477-87.

32. Mendell JR, Goemans N, Lowes LP, et al. Longitudinal effect of eteplirsen versus historical control on ambulation in Duchenne muscular dystrophy. Ann Neurol. 2016;79(2):257-71.

33. Bushby K, Finkel R, Birnkrant DJ, et al. Diagnosis and management of Duchenne muscular dystrophy, part 2: implementation of multidisciplinary care. Lancet Neurol. 2010;9(2):177-89.

34. Access Economics. The cost of muscular dystrophy. Muscular Dystrophy Australia. October 19, 2007. Available at: http://www.mda.org.au/media/ accesslaunch/. Accessed April 27, 2017. 


\section{APPENDIX Codes Identifying Respiratory Failure, Respiratory Therapy, and Ventilation}

\begin{tabular}{|c|c|c|}
\hline Code Type & Codes & Description \\
\hline \multirow{7}{*}{$\begin{array}{l}\mathrm{ICD}-9-\mathrm{CM} \\
\text { procedure }\end{array}$} & 89.38 & Other nonoperative respiratory measurements \\
\hline & 93.90 & Noninvasive mechanical ventilation \\
\hline & 93.94 & Respiratory medication administered by nebulizer \\
\hline & 93.99 & Other respiratory therapy \\
\hline & 96.70 & Continuous invasive mechanical ventilation of unspecified duration \\
\hline & 96.71 & Continuous invasive mechanical ventilation for less than 96 consecutive hours \\
\hline & 96.72 & Continuous invasive mechanical ventilation for 96 consecutive hours or more \\
\hline \multirow{8}{*}{$\begin{array}{l}\text { ICD-9-CM } \\
\text { diagnosis }\end{array}$} & 518.81 & Acute respiratory failure \\
\hline & 518.82 & Other pulmonary insufficiency, not elsewhere classified \\
\hline & 518.83 & Chronic respiratory failure \\
\hline & 518.84 & Acute and chronic respiratory failure \\
\hline & V46.11 & Dependence on respirator, status \\
\hline & V46.12 & Encounter for respirator dependence during power failure \\
\hline & V46.13 & Encounter for weaning from respirator [ventilator] \\
\hline & V46.14 & Mechanical complication of respirator [ventilator] \\
\hline \multirow[t]{26}{*}{ CPT/HCPCS } & 94002 & $\begin{array}{l}\text { Ventilation assist and management, initiation of pressure or volume preset ventilators for assisted or controlled breathing; hospital } \\
\text { inpatient/observation, initial day }\end{array}$ \\
\hline & 94003 & $\begin{array}{l}\text { Ventilation assist and management, initiation of pressure or volume preset ventilators for assisted or controlled breathing; hospital } \\
\text { inpatient/observation, each subsequent day }\end{array}$ \\
\hline & 94004 & $\begin{array}{l}\text { Ventilation assist and management, initiation of pressure or volume preset ventilators for assisted or controlled breathing; nursing } \\
\text { facility, per day }\end{array}$ \\
\hline & 94005 & $\begin{array}{l}\text { Home ventilator management care plan oversight of a patient (patient not present) in home, domiciliary or rest home } \\
\text { (e.g., assisted living) requiring review of status, review of laboratories and other studies and revision of orders and respiratory } \\
\text { care plan (as appropriate), within a calendar month, } 30 \text { minutes or more }\end{array}$ \\
\hline & 94644 & Continuous inhalation treatment with aerosol medication for acute airway obstruction; first hour \\
\hline & 94645 & $\begin{array}{l}\text { Continuous inhalation treatment with aerosol medication for acute airway obstruction; each additional hour (list separately in } \\
\text { addition to code for primary procedure) }\end{array}$ \\
\hline & 94650 & Pressure breathing (intermittent positive pressure breathing), initial \\
\hline & 94651 & Pressure breathing (intermittent positive pressure breathing), subsequent \\
\hline & 94652 & Pressure breathing (intermittent positive pressure breathing), newborn infants \\
\hline & 94656 & $\begin{array}{l}\text { Ventilation assist and management, initiation of pressure or volume preset ventilators for assisted or controlled breathing; } \\
\text { first day }\end{array}$ \\
\hline & 94657 & $\begin{array}{l}\text { Ventilation assist and management, initiation of pressure or volume preset ventilators for assisted or controlled breathing; } \\
\text { subsequent days }\end{array}$ \\
\hline & 94660 & Continuous positive airway pressure ventilation, initiation and management \\
\hline & 94662 & Continuous negative pressure ventilation, initiation and management \\
\hline & 94667 & $\begin{array}{l}\text { Manipulation chest wall, such as cupping, percussing, and vibration to facilitate lung function; initial demonstration and/or } \\
\text { evaluation }\end{array}$ \\
\hline & 94668 & Manipulation chest wall, such as cupping, percussing, and vibration to facilitate lung function; subsequent \\
\hline & 99504 & Home visit for mechanical ventilation care \\
\hline & E0450 & $\begin{array}{l}\text { Volume control ventilator, without pressure support mode, may include pressure control mode, used with invasive interface } \\
\text { (e.g., tracheostomy tube) }\end{array}$ \\
\hline & E0453 & Therapeutic ventilator; suitable for use 12 hours or less per day \\
\hline & E0454 & Pressure ventilator with pressure control \\
\hline & E0460 & Negative pressure ventilator; portable or stationary \\
\hline & E0461 & $\begin{array}{l}\text { Volume control ventilator, without pressure support mode, may include pressure control mode, used with noninvasive interface } \\
\text { (e.g., mask) }\end{array}$ \\
\hline & E0463 & $\begin{array}{l}\text { Pressure support ventilator with volume control mode, may include pressure control mode, used with invasive interface } \\
\text { (e.g., tracheostomy tube) }\end{array}$ \\
\hline & E0464 & $\begin{array}{l}\text { Pressure support ventilator with volume control mode, may include pressure control mode, used with noninvasive interface } \\
\text { (e.g., mask) }\end{array}$ \\
\hline & E0470 & $\begin{array}{l}\text { Respiratory assist device, bi-level pressure capability, without back-up rate feature, used with noninvasive interface, e.g., nasal or } \\
\text { facial mask (intermittent assist device with continuous positive airway pressure device) }\end{array}$ \\
\hline & E0471 & $\begin{array}{l}\text { Respiratory assist device, bi-level pressure capability, with back-up rate feature, used with noninvasive interface, e.g., nasal or } \\
\text { facial mask (intermittent assist device with continuous positive airway pressure device) }\end{array}$ \\
\hline & E0472 & $\begin{array}{l}\text { Respiratory assist device, bi-level pressure capability, with back-up rate feature, used with invasive interface, e.g., tracheostomy } \\
\text { tube (intermittent assist device with continuous positive airway pressure device) }\end{array}$ \\
\hline
\end{tabular}


The Direct Cost of Managing a Rare Disease: Assessing Medical and

Pharmacy Costs Associated with Duchenne Muscular Dystrophy in the United States

\section{APPENDIX Codes Identifying Respiratory Failure, Respiratory Therapy, and Ventilation (continued)}

\begin{tabular}{|c|c|c|}
\hline Code Type & Codes & Description \\
\hline \multirow[t]{15}{*}{$\overline{\mathrm{CPT} / \mathrm{HCPCS}}$} & E0480 & Percussor, electric or pneumatic, home model \\
\hline & E0481 & Intrapulmonary percussive ventilation system and related accessories \\
\hline & E0482 & Cough stimulating device, alternating positive and negative airway pressure \\
\hline & E0483 & High frequency chest wall oscillation air-pulse generator system (includes hoses and vest), each \\
\hline & E0484 & Oscillatory positive expiratory pressure device, nonelectric, any type, each \\
\hline & E0485 & $\begin{array}{l}\text { Oral device/appliance used to reduce upper airway collapsibility, adjustable or nonadjustable, prefabricated, includes fitting and } \\
\text { adjustment }\end{array}$ \\
\hline & E0486 & $\begin{array}{l}\text { Oral device/appliance used to reduce upper airway collapsibility, adjustable or nonadjustable, custom fabricated, includes fitting } \\
\text { and adjustment }\end{array}$ \\
\hline & E0500 & $\begin{array}{l}\text { Intermittent positive pressure breathing machine, all types, with built-in nebulization; manual or automatic valves; internal or } \\
\text { external power source }\end{array}$ \\
\hline & G0237 & $\begin{array}{l}\text { Therapeutic procedures to increase strength or endurance of respiratory muscles, face to face, one on one, each } 15 \text { minutes } \\
\text { (includes monitoring) }\end{array}$ \\
\hline & G0238 & $\begin{array}{l}\text { Therapeutic procedures to improve respiratory function, other than described by G0237, one on one, face to face, per } 15 \text { minutes } \\
\text { (includes monitoring) }\end{array}$ \\
\hline & G0239 & $\begin{array}{l}\text { Therapeutic procedures to improve respiratory function or increase strength or endurance of respiratory muscles, } 2 \text { or more } \\
\text { individuals (includes monitoring) }\end{array}$ \\
\hline & G0424 & Pulmonary rehabilitation, including exercise (includes monitoring), 1 hour, per session, up to 2 sessions per day \\
\hline & S5180 & Home health respiratory therapy, initial evaluation \\
\hline & S5181 & Home health respiratory therapy, not otherwise specified, per diem \\
\hline & S9473 & Pulmonary rehabilitation program, nonphysician provider, per diem \\
\hline
\end{tabular}

CPT = Current Procedural Terminology; HCPCS = Healthcare Common Procedure Coding System; ICD-9-CM = International Classification of Diseases, Ninth Revision, Clinical Modification. 